\title{
An Improved Technique of Channel Estimation for OFDM in DVB-T2
}

\author{
Ahmed H.Eldieb \\ Electronics and \\ Communications Eng. Dept., \\ Higher Institute of Engineering, \\ Modern Academy \\ Cairo, Egypt
}

\author{
Mona Z.Saleh \\ Electronics and \\ Communications Eng. Dept., \\ Faculty of Engineering, \\ Ain Shams University \\ Cairo, Egypt
}

\author{
Salwa Elramly \\ Electronics and \\ Communications Eng. Dept., \\ Faculty of Engineering, \\ Ain Shams University \\ Cairo, Egypt
}

\begin{abstract}
Orthogonal Frequency Division Multiplexing (OFDM) with pilot assisted channel estimation is widely applied in wireless communication systems due to its high rate transmission capability and its robustness to multi-path fading and delay. In this paper an improved channel estimation method for OFDM in second generation Digital Video Broadcasting for Terrestrial (DVB-T2) is presented based on a pilot arrangement in time-varying frequency-selective fading channels. A new method which is based on an improved Domain Transform Least Square Estimation method (DTLSE) and a Two Dimensional Interpolation method (2-DI) have been proposed. The proposed methods give a good BER performance compared to other considered methods .The estimation matrix size for obtaining Channel Impulse Response (CIR) depends only on the length of the channel not on the number of pilot subcarriers nor the size of OFDM symbol, it does not require knowledge of channel statistics and works for any pilot pattern. The Minimum Mean Squared Error (MMSE) channel estimation was considered as the reference method that gives the best BER performance but suffers from a high computational load. The proposed method is compared with the two other methods: the improved DTLSE and MMSE. The proposed method proves to give a reduction in complexity compared to the MMSE technique with a comparable performance but better than the DTLSE one.
\end{abstract}

\section{General Terms}

Pilot-assisted channel estimation, wireless systems, DVB-T2.

\section{Keywords}

The second generation for Digital Video Broadcasting Terrestrial system (DVB-T2), improved Domain Transform Least Square Estimation (DTLSE), two dimensional Interpolation (2-DI), Minimum Mean Square Estimation (MMSE).

\section{INTRODUCTION}

OFDM is a well-known multicarrier technique which provides an effective and low complexity means of reducing intersymbol interference. Due to its high bandwidth efficiency high transmission capability and its robustness against multipath delay, it has been widely applied in wireless communication systems for transmissions over frequencyselective fading channels [1]. OFDM has been used in many standards such as Digital Audio Broadcasting (DAB);Digital television DVB-T/T2 (terrestrial) [2]; Wireless LAN IEEE 802.11a, IEEE 802.11g; IEEE 802.11n;the LTE, LTE Advanced and $4 \mathrm{G}$ mobile phone standards.
The Inverse Fast Fourier Transform (IFFT) is used for modulating the data constellations on the orthogonal subcarriers at the OFDM system transmitter side. These constellations can be taken according to an M-ary PSK or QAM modulation. A guard interval is inserted to prevent inter-symbol interference. The Digital to Analog Converter (DAC) is applied for transmitting the signal on channel [3].

At the receiver side, after removing the guard interval, demodulation is performed by Fast Fourier Transform (FFT). Channel estimation is required in order to retrieve the data contained in these signal constellations and compensate the channel effects such as attenuation distortion, delay distortion and noise. Channel est-imation is usually performed by transmitting pilot symbols on subcarriers where these pilots are known to the receiver [4]. Many estimation methods are found in the literature; here some of them are cited.

Channel estimation in frequency-domain pilot assisted OFDM systems can be obtained by employing the time-domain sequence of modulated pilot subcarriers to estimate the Channel Impulse Response (CIR) which is known as the Time Domain Least Square Estimation (TDLSE) technique [5]. Another process which employing the LS estimate of pilot subchannels to estimate time-domain channel impulse response (CIR) and then transforming it to Channel Transfer Function (CTF) which is known as the domain-transform method (DTLSE) [6]. The last method is employing the leastsquares (LS) estimate of pilot subchannels to estimate the complete frequency-domain CTF which is known Frequency Domain Least Square Estimation (FDLSE). Among these methods the MMSE estimation [7] offers the best BER but has heavy computational load and needs knowledge of channel statistics.

The proposed estimation method which is based on an improved (DTLSE) and (2-DI) methods gives better performance than the improved DTLSE and reduces the computational complexity than the MMSE method. The estimation matrix for obtaining CIR depends only on the length of the channel not the number of pilot subcarriers or the size of OFDM symbol. It also works for any frequency pilot pattern without needing prior knowledge of channel statistics.

The paper is organized as follows; the system model and principle of channel estimation are explained in section (2); performance analysis of the proposed and other methods is presented in section (3); computational load of the considered methods are calculated in section (4); the simulation results are presented in section (5) while section (6) concludes the paper. 


\section{SYSTEM MODEL}

A general baseband OFDM system is used over a frequencyselective fading channel through a single transmitter and receiver antenna. In each OFDM symbol, there are $\mathrm{N}$ subcarriers independently. The frequency -domain signal X (k) is the FFT of the discrete baseband $\mathrm{x}(\mathrm{n})$ as

$$
X(k)=\sum_{n=0}^{N-1} x(n) e^{-i \frac{2 \pi n k}{N}}
$$

where the subcarrier index $\mathrm{k}=0, \ldots, \mathrm{K}-1$ and $\mathrm{K}$ is the total number of subcarriers. In this case the channel can be viewed as a set of parallel subchannels and the received signal is represented as

$$
\mathrm{Y}(\mathrm{k})=\mathrm{H}(\mathrm{k}) \mathrm{X}(\mathrm{k})+\mathrm{W}(\mathrm{k})
$$

where $\mathrm{H}(\mathrm{k})$ and $\mathrm{W}(\mathrm{k})$ are the CFR and the AWGN, respectively. Channel estimation method finds the channel estimate $\widehat{\mathrm{H}}\left(\mathrm{k}_{\mathrm{p}}\right)$. This is obtained by comparing the received pilot subcarriers with the transmitted one which are known to the receiver, where $K_{P}$ is the total number of pilots and $k_{P}$ denotes the index of the pilot subcarrier [8],[9]. The estimation of CFR at a pilot position can be obtained by the LS method as

$$
\widehat{\mathrm{K}}\left(\mathrm{k}_{\mathrm{p}}\right)=\frac{Y\left(\mathrm{k}_{\mathrm{p}}\right)}{X\left(\mathrm{k}_{\mathrm{p}}\right)}
$$

where $\mathrm{Y}\left(\mathrm{k}_{\mathrm{p}}\right)$ and $\mathrm{X}\left(\mathrm{k}_{\mathrm{p}}\right)$ are the received pilot subcarrier and the transmitted one respectively. The CFR values at the rest of the subcarriers are calculated through linear, polynomial or spline interpolation. The most common interpolation method is the linear one because of its simplicity and low computational complexity [10].

In DVB-T2 system for pilot pattern (PP2), the scattered pilots used for channel estimation are spread in both time and frequency directions. As shown in Figure 1, there is one pilot subcarrier out of six subcarriers in the frequency direction and one pilot out of two symbols in time direction. Thus the pilot density for this pilot pattern is $D_{x}=6$ and $D_{y}=2$ plus edge

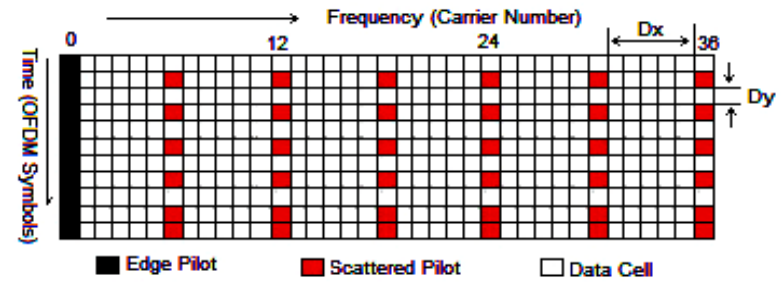

pilots, where $D_{x}$ is the Difference in carrier index between adjacent scattered-pilot-bearing carriers and $\mathrm{D}_{\mathrm{y}}$ is the Difference in symbol number between successive scattered pilots on a given carrier [11].

Fig 1: Scattered pilot pattern (PP2) [2].

In the following section the performance analysis of the proposed method compared with other methods will be introduced.

\section{PERFORMANCE ANALYSIS OF THE PROPOSED AND PUBLISHED METHODS}

In this section, three channel estimation methods are presented. Two of them are already published in the literature while the third one is a new proposed algorithm which has better performance in BER than the others

\subsection{Improved Domain Transform Estimation Method}

In the IDTE method [12], the least square estimation of pilot subchannels is employed to estimate time-domain CIR and then transform it to CTF. The CIR is then given as [12]:

$$
\hat{h}_{L}=\left(\frac{1}{k_{P}}\right) \mathrm{I}_{\mathrm{L}} \cdot \mathrm{F}_{k_{P}, \mathrm{~L}}^{\mathrm{H}} \cdot \mathrm{D}\left(\mathrm{X}_{k_{P}}\right)^{\mathrm{H}} \cdot \mathrm{Y}_{k_{P}}
$$

where $\hat{h}_{L}$ is the $\mathrm{CIR}, \mathrm{F}_{k_{P}, \mathrm{~L}}^{\mathrm{H}}$ is a $L \times K_{P}$ Fourier transform coefficients matrix, $(.)^{\mathrm{H}}$ denotes Hermitian transpose, $\mathrm{D}\left(\mathrm{X}_{k_{P}}\right)$ is a diagonal matrix with $\mathrm{X}_{k_{P}}$ on its diagonal, $\mathrm{I}$ is the $L \times$ $L$ identity matrix and $\mathrm{L}$ is the length of the channel. In addition to avoiding the use of matrix inversion, the size of the estimation matrix $\hat{h}_{L}$ for calculating CIR depends only on the length of the channel.

\subsection{The MMSE Estimation Method}

The MMSE estimation [7], provides the best BER performance. It estimates CTF $\widehat{\mathrm{H}}_{\text {mmse }}$ as

$$
\widehat{\mathrm{H}}_{\text {mmse }}=\mathrm{R}_{k, k_{p}} \cdot \mathrm{R}_{k_{p}, k_{p}}^{-1 / 2} \cdot \mathrm{R}_{k_{p}, k_{p}}^{-1 / 2} \cdot \widehat{\mathrm{H}}_{k_{p}, \mathrm{ls}}
$$

where $\mathrm{R}_{k, k_{P}}$ of size $K \times K_{P}$ is the correlation matrix of complete CTF with pilot subchannels, and $\mathrm{R}_{k_{P}, k_{P}}$ of size $K_{P} \times K_{P}$ is the correlation matrix of pilot subchannels with its LS estimate $\widehat{\mathrm{H}}_{k_{p}, \mathrm{ls}}$. This method suffers from heavy computional load and the need of channel statistics prior knowledge.

\subsection{The proposed Method}

For slow fading channels, it can be assumed that the channel coefficients at a subcarrier throughout several OFDM symbols remain approximately constant [8], [10].Therefore, pilot subcarrier spacing in the frequency direction is reduced from twelve to six. Based on (3) the two Dimensional estimation matrix of CFR can be obtained by the LS method as

$$
\widehat{\mathrm{H}}_{\mathrm{L}, \mathrm{L}}=\mathrm{X}_{\mathrm{L}, \mathrm{k}_{\mathrm{P}}}^{-1}, \mathrm{Y}_{\mathrm{k}_{\mathrm{P}, \mathrm{L}}}
$$

where $\widehat{\mathrm{H}}_{\mathrm{L}, \mathrm{L}}$ is $L \times L$ CFR estimation matrix, $\mathrm{X}_{\mathrm{L}, \mathrm{k}_{\mathrm{P}}}^{-1}$ is $L \times K_{P}$ transmitted matrix and $\mathrm{Y}_{\mathrm{k}_{\mathrm{P}} \mathrm{L}}$ is $K_{P} \times L$ received matrix. By applying least square solution the CFR estimation matrix is given as

$$
\widehat{\mathrm{H}}_{\mathrm{L}, \mathrm{L}}=\left(\mathrm{X}_{\mathrm{k}_{\mathrm{P}, \mathrm{L}}^{\mathrm{T}}} \cdot \mathrm{X}_{\mathrm{k}_{\mathrm{P}, \mathrm{L}}}\right)^{-1} \cdot \mathrm{X}_{\mathrm{k}_{\mathrm{P}, \mathrm{L}}^{\mathrm{T}}}^{\mathrm{T}} \cdot \mathrm{Y}_{\mathrm{k}_{\mathrm{P}, \mathrm{L}}}
$$

The noise in $\widehat{H}_{\mathrm{L}, \mathrm{L}}$ will be severely amplified by $\mathrm{X}^{-1}$, leading to an estimate of CFR far away from its actual value. This problem can be solved by Tikhonov regularization [13] as

$$
\widehat{\mathrm{H}}_{\mathrm{L}, \mathrm{L}}=\left(\mathrm{X}_{\mathrm{k}_{\mathrm{P}, \mathrm{L}}}^{\mathrm{T}} \cdot \mathrm{X}_{\mathrm{k}_{\mathrm{P}, \mathrm{L}}}+\alpha \mathrm{I}_{\mathrm{L}, \mathrm{L}}\right)^{-1} \cdot \mathrm{X}_{\mathrm{k}_{\mathrm{P}, \mathrm{L}}}^{\mathrm{T}} \cdot \mathrm{Y}_{\mathrm{k}_{\mathrm{P}, \mathrm{L}}}
$$

where $\mathrm{I}$ is the $\mathrm{L} \times \mathrm{L}$ identity matrix and $\alpha$ is a regularization parameter. The choice of $\alpha$ can be obtained using two methods, the L-curve method [13] and the strategy proposed in [14] as will be explained in section 3.3.1 and 3.3.2

Based on (4) the CIR is given as 


$$
\widehat{\mathrm{h}}_{\mathrm{L}, \mathrm{L}}=\left(\frac{1}{\mathrm{k}_{\mathrm{P}}}\right) \mathrm{I}_{\mathrm{L}, \mathrm{L}} \cdot \mathrm{F}_{\mathrm{L}, \mathrm{L}}^{\mathrm{H}} \cdot \widehat{\mathrm{H}}_{\mathrm{L}, \mathrm{L}}
$$

So, from (8) and (9) the CIR is then given as

$$
\hat{\mathrm{h}}_{\mathrm{L}, \mathrm{L}}=\left(\frac{1}{\mathrm{k}_{\mathrm{P}}}\right) \mathrm{I}_{\mathrm{L}, \mathrm{L}} \cdot \mathrm{F}_{\mathrm{L}, \mathrm{L}}^{\mathrm{H}} \cdot\left(\left(\mathrm{X}_{\mathrm{k}_{\mathrm{P}, \mathrm{L}}^{\mathrm{T}}}^{\mathrm{T}} \cdot \mathrm{X}_{\mathrm{k}_{\mathrm{P}, \mathrm{L}}}+\alpha \mathrm{I}_{\mathrm{L}, \mathrm{L}}\right)^{-1} \cdot \mathrm{X}_{\mathrm{k}_{\mathrm{P}, \mathrm{L}}^{\mathrm{T}}}^{\mathrm{T}} \cdot \mathrm{Y}_{\mathrm{k}_{\mathrm{P}}, \mathrm{L}}\right)
$$

Based on (10) the size of the estimation matrix $\hat{h}_{\mathrm{L}, \mathrm{L}}$ for obtaining CIR depends only on the length of the channel. The whole effective CTF is estimated by applying N-point FFT on the zero padded estimated effective CIR.

In the following subsection some strategies to choose $\alpha$ are shown.

\begin{tabular}{|c|c|c|c|c|c|}
\hline \multicolumn{2}{|c|}{$\begin{array}{c}\text { TU6 } \\
\text { Channel }\end{array}$} & \multicolumn{2}{c|}{$\begin{array}{c}\text { Brazil } \\
\text { Channel D }\end{array}$} & \multicolumn{2}{c|}{$\begin{array}{c}\text { high delay } \\
\text { Rayleigh fading } \\
\text { Channel }\end{array}$} \\
\hline $\mathrm{D}$ & $\mathrm{P}(\mathrm{dB})$ & $\mathrm{D}$ & $\mathrm{P}(\mathrm{dB})$ & $\mathrm{D}$ & $\mathrm{P}(\mathrm{dB})$ \\
\hline 0 & -3 & 0 & -0.1 & 0 & 0 \\
\hline 2 & 0 & 5 & -3.9 & 2 & -5 \\
\hline 5 & -2 & 20 & -2.6 & 17 & -7 \\
\hline 15 & -6 & 29 & -1.3 & 36 & -8.8 \\
\hline 21 & -8 & 57 & 0 & 75 & -10 \\
\hline 46 & -10 & 58 & -2.8 & 137 & -10 \\
\hline
\end{tabular}

\subsubsection{L-curve method}

It plots $E\left[e_{r}\right]$ and $E\left[e_{n}\right]$ under different $\alpha^{\prime} \mathrm{s}$ where $\mathrm{E}$ is the expectation operator, $e_{r}$ and $e_{n}$ are regularization error and additive noise error respectively, to graphically search a tradeoff point, which minimizes $E\left[e_{r}\right]+E\left[e_{n}\right]$ [13].Base on (8),

$$
\widehat{\mathrm{H}}_{\mathrm{L}, \mathrm{L}}=\mathrm{A} \mathrm{Y}_{\mathrm{k}_{\mathrm{P}, \mathrm{L}}}
$$

Minimizing the estimation error $\mathrm{e}=\left\|\mathrm{A} . \mathrm{Y}_{\mathrm{k}_{\mathrm{P}, \mathrm{L}}}-\mathrm{H}_{\mathrm{L}, \mathrm{L}}\right\|$ highly depends on choosing a proper regularization parameter $\alpha$. The estimation error $e$ is decomposed into $e_{r}$ and $e_{n}$.

$$
\begin{aligned}
\mathrm{e} & =\left\|\mathrm{A} \cdot \mathrm{Y}_{\mathrm{k}_{\mathrm{P}, \mathrm{L}}}-\mathrm{H}_{\mathrm{L}, \mathrm{L}}\right\| \\
& =\left\|\mathrm{A} \cdot\left(\mathrm{Y}_{\mathrm{k}_{\mathrm{P}, \mathrm{L}}}+\mathrm{W}\right)-\mathrm{X}_{\mathrm{L}, \mathrm{k}_{\mathrm{P}},}^{-1} \cdot \mathrm{Y}_{\mathrm{k}_{\mathrm{P}, \mathrm{L}}}\right\| \\
\leq & \left\|\left(\mathrm{A}-\mathrm{X}_{\mathrm{L}, \mathrm{k}_{\mathrm{P}},}^{-1}\right) \cdot \mathrm{Y}_{\mathrm{k}_{\mathrm{P}, \mathrm{L}}}\right\|+\|\mathrm{A} \cdot \mathrm{W}\|=e_{r}+e_{n}
\end{aligned}
$$

One strategy to balance these two errors is called the L-curve method [13].as shown in Figure 2.

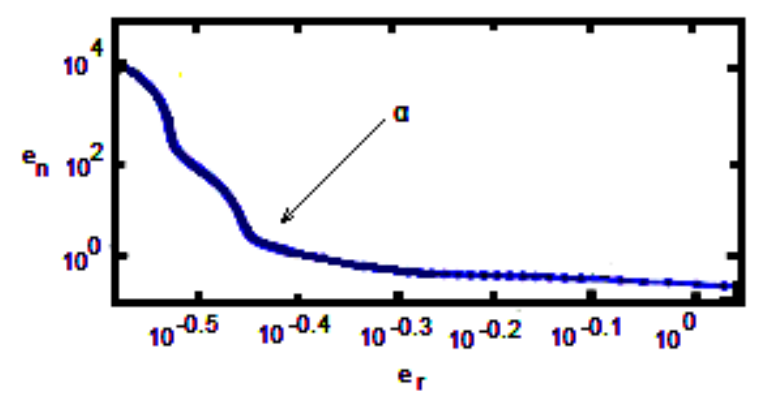

Fig 2: The L-curve method to choose regularization parameter $\alpha[13]$.

\subsubsection{Mathematical Method}

Another strategy proposed in [14] is used in which the expression of $\alpha$ given for pilots amplitude-boosted $(\beta)$ and $\operatorname{SNR}\left(\gamma^{2}\right)$ as

$$
\alpha=\left(\frac{\sqrt{K_{\mathrm{P}}}}{\beta \gamma}\right)^{2 / 3}
$$

\section{COMPUTATIONAL LOAD OF THE DISCUSSED METHODS}

The computational load or the amount of arithmetic operations is an important factor for distinguishing between the channel estimation methods [12]. In Strassen algorithm [15] the multiplication between an $A_{1} \times A_{2}$ matrix and an $A_{2} \times A_{3}$ matrix requires about $2 \mathrm{~A}_{1} \mathrm{~A}_{2} \mathrm{~A}_{3}$ arithmetic operations and the Inverse of an $\mathrm{A} \times \mathrm{A}$ matrix requires about $5.5 \mathrm{~A}^{2.8}$ arithmetic operations. The number of subcarriers and pilots are denoted by $\mathrm{N}, \mathrm{N}_{\mathrm{p}}$ respectively while the ratio of them is defined as $\rho=N_{p} / N$ and $L$ is the length of channel. The comparison between the proposed and other methods in terms of computational load is shown in Figure 3.

1) For Improved Domain Transform Estimation Method, computation of (6) requires: $2 \mathrm{~L} \rho^{2} \mathrm{~N}^{2}+2 \mathrm{~L} \rho \mathrm{N}$ arithmetic operations [12].

2) For MMSE, computation of (5) requires: $4 \rho^{2} \mathrm{~N}^{3}+$ $5.5 \rho^{2.8} \mathrm{~N}^{2.8}+3 \rho \mathrm{N}^{2}$ arithmetic operations [7].

$3)$ For the proposed Method, computation of (12) requires: $2\left(\left(\rho^{3} \mathrm{~N}^{3}+\mathrm{L}^{3}+\rho \mathrm{N}^{2}(1+\rho \mathrm{L})\right)+\rho \mathrm{N}(1+2 \mathrm{~L})+\right.$ $5.5 \rho^{2.8} \mathrm{~N}^{2.8}$ arithmetic operations.

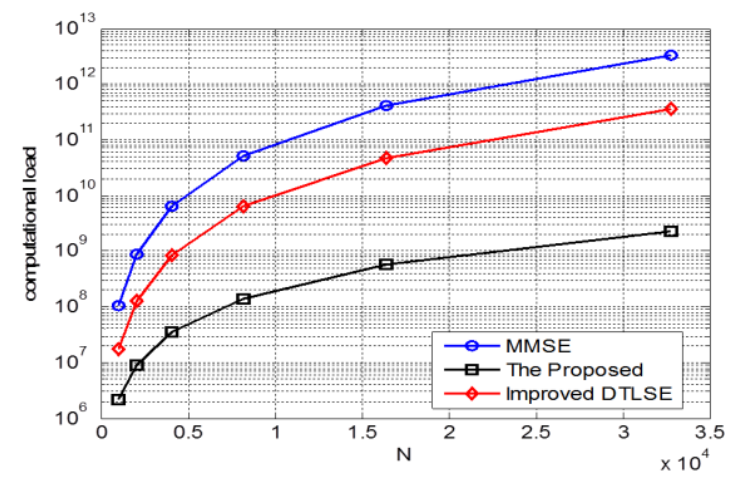

Fig 3: The computational load for the proposed and other methods

\section{SIMULATION RESULTS}

The simulated OFDM system is adopted from DVB-T2 [2], with a bandwidth of $8 \mathrm{MHz}$. There are 1024 sub-carriers, data subcarriers are QPSK modulated. Pilot pattern parameters are $D_{x}=6$ and $D_{y}=2$ plus edge pilots. The CP length is 64 and the amplitude is boosted by a factor of $\beta=1.33$. Three channel models were investigated, TU-6 channel [2], a Brazil Channel D [16] and high delay spread Rayleigh fading channel [2] with power delay profiles for relative path delay (D) and power $(\mathrm{P} \mathrm{dB})$ given in Table I. The BER performance of the proposed method was simulated and compared with Improved Domain Transform Estimation and MMSE methods.

Table 1. Power delay profiles 
Under TU6 channel conditions the proposed method provides BER values $1 \mathrm{~dB}$ higher than that with perfect channel estimation and $1.5 \mathrm{~dB}$ less than the improved DTLSE. This difference is negligible for the MMSE method as shown in figure 4. In case of a Brazil channel [16], as shown in figure 5 the BER of the proposed and the other methods are slightly higher than those in Fig.4. This means that Brazil channel is more frequency selective than TU-6 channel.

In high delay spread Rayleigh fading channel [2], the proposed and the other methods are severely higher than that in Fig.4 and Fig.5 as shown in Fig. 6. This means that the Rayleigh fading channel has a root-mean-square delay spread higher than TU-6 channel and Brazil channel.

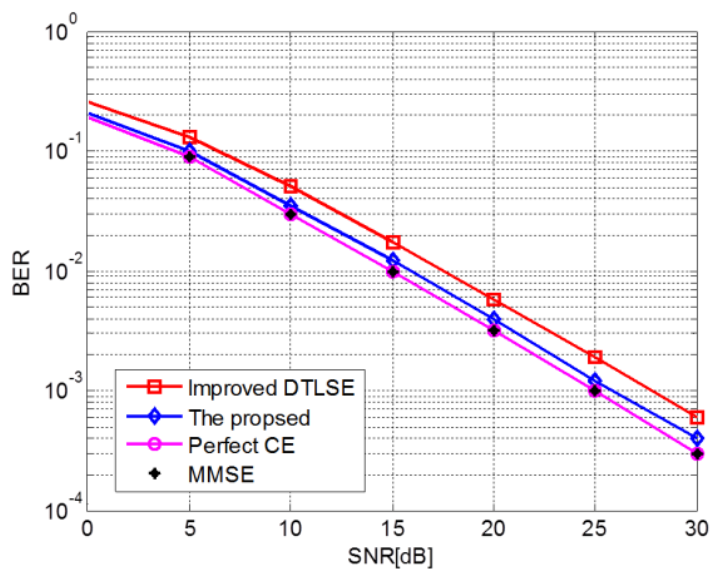

Fig 4: BER vs. SNR for the proposed and other methods in TU-6 Channel.

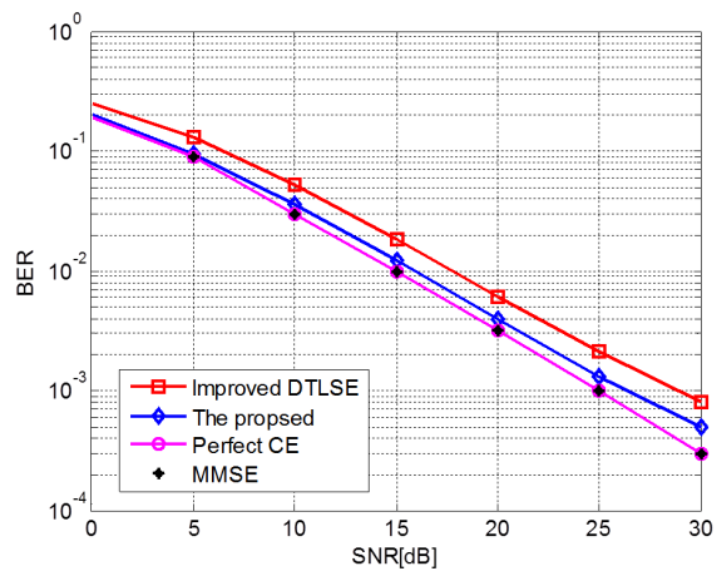

Fig 5: BER vs. SNR for the proposed and other methods in Brazil Channel D

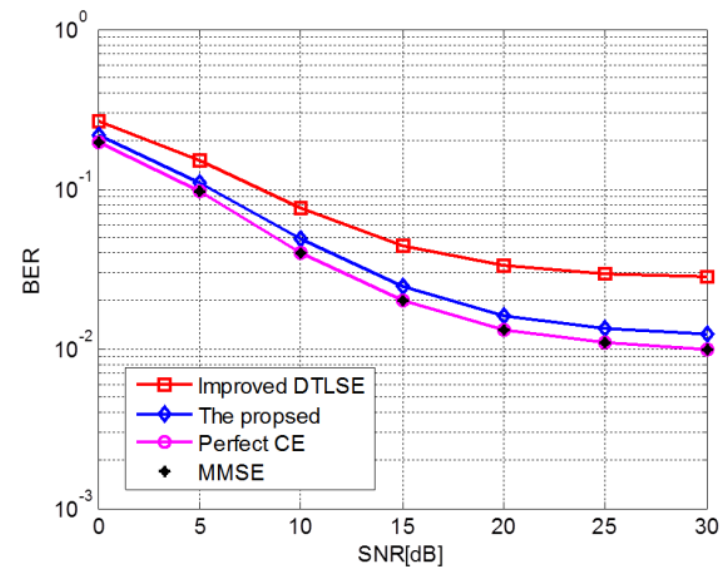

Fig 6: BER vs. SNR for the proposed and other methods in high delay Rayleigh fading Channel

\section{CONCULSION}

A modified method based on improved Domain Transform Least Square Estimation method (DTLSE) and a Two Domain Interpolation method (2-DI) has been proposed gives a good performance in BER than the other considered method. The size of the estimation matrix for obtaining CIR depends only on the length of the channel not on the number of pilot subcarriers or the size of OFDM symbol. It also works for any frequency pilot pattern and it does not require knowledge of channel statistics. The proposed method performance is close to MMSE and better than the DTLSE method and still has a reduction in complexity compared to the MMSE technique. The proposed method provides BER values $1 \mathrm{~dB}$ higher than that with MMSE and $1.5 \mathrm{~dB}$ less than the improved DTLSE, but the computational load of MMSE compared to the proposed method for a $1 \mathrm{k}$ DVB-T2 mode is 7:1 bigger.

\section{RERFERNCES}

[1] R. Prasad, 2004. OFDM for Wireless Communications Systems, Singapore, Artech House.

[2] DVB Document A133, February, 2012. Implementation guidelines for a second generation Digital terrestrial television broadcasting system (DVB-T2)

[3] K. Witrisal, "OFDM Air-Interface Design for Multimedia Communications", Ch. 4, Ph.D. Thesis, Delft University of Technology, The Netherlands, April 2002

[4] Yong Soo Cho, Jackson Kim,Won Young Yang,ChungGu Kang,"MIMO-OFDM wireless communications with MALAB," John Wiley \& Sons (Asia) Pte Ltd., 2010.

[5] J. Lin, "Least-squares channel estimation for mobile OFDM communication on time-varying frequencyselective fading channels," IEEE Trans.Veh. Technol., vol. 57, no. 6, pp. 3538-3550, Nov. 2008.

[6] Mingchao Yu and Parastoo Sadeghi," Study of PilotAssisted OFDM Channel Estimation Methods with Improvements for DVB-T2," IEEE Trans.Vehicular Technology, June 2012, Vol. 61, No. 5.

[7] O. Edfors, M. Sandell, J.-J. Van de Beek, S. Wilson, and P. Borjesson, "OFDM channel estimation by singular value decomposition,'IEEE Trans. Commun., Jul. 1998, Vol. 46, No. 7, pp. 931-939.

[8] D. C. Chang, "Effect and compensation of symbol timing offset in OFDM systems with channel 
interpolation," IEEE Trans. Broadcast., Dec. 2008, Vol. 54, No. 4, pp. 761-770.

[9] J. Park, J. Kim, M. Park, K. Ko, C. Kang, and D. Hong, "Performance analysis of channel estimation for OFDM systems with residual timing offset," IEEE Trans. Wireless Commun.,July 2006,Vol. 5, no. 7, pp. 16221625.

[10] Y.S. Lee, H.N. Kim,S. I. Park, and S. I. Lee, "Noise reduction for channel estimation based on pilot-block averaging in DVB-T receivers,"IEEE Trans. Consumer Electron., Feb. 2006, Vol. 52, no. 1, pp. 51-58.

[11] DVB Document A122 Frame structure channel coding and modulation for a second generation digital terrestrial television broadcasting system (DVB-T2), June. 2008.
[12] Ahmed H.Eldieb, Mona Z.Saleh and Salwa Elramly," A Comparative Study of Channel Estimation Techniques for OFDM in DVB-T2," IJCA (0975 - 8887), April 2014, Volume 91 - No.14.

[13] P. C. Hansen, Rank-Deficient and Discrete Ill-Posed Problems.Philadelphia, PA: SIAM, 1998.

[14] M. T. Nair, Linear Operator Equations: Approximation and Regularization. Singapore: World Scientific, 2009.

[15] T. H. Cormen, C. E. Leiserson, R. L. Rivest, and C. Stein, Introduction to Algorithms. Cambridge, MA: MIT Press, 2001

[16] Mackenzie, ABERT, SET, "General description of laboratory tests," DTV Field Test Report in Brazil, July 2000 . 